Revista Brasileira de Agricultura Irrigada v.14, nº.2, p. 3987 - 3996, 2020

ISSN 1982-7679 (On-line)

Fortaleza, CE, INOVAGRI - http://www.inovagri.org.br

DOI: $10.7127 /$ rbai.v14n101154

Protocolo 1154.20 - 25/04/2020 Aprovado em 06/06/2020

\title{
AVALIAÇÃO DE MÉTODO ALTERNATIVO E DE CONDUTIVÍMETROS NA DETERMINAÇÃO DA CONDUTIVIDADE ELÉTRICA DO SOLO
}

Tonny José Araújo da Silva ${ }^{1}$, Matheus Nogueira Fonseca dos Santos ${ }^{2}$, Adriano Bicioni Pacheco ${ }^{3}$, Edna Maria Bonfim-Silva ${ }^{4}$, Thiago Franco Duarte ${ }^{5}$

\section{RESUMO}

Objetivou-se avaliar, por meio de índices estatísticos, a estimativa da condutividade elétrica do solo pela metodologia relação 1:1 (terra: água destila) e condutivímetros alternativos, em um Latossolo Vermelho distrófico. O solo foi submetido ao processo de salinização, com diferentes concentrações de cloreto de potássio. Foi adotada como referência a condutividade elétrica do solo do extrato de solução, mensurada pelo condutivímetro mCA 150 Tecnal $^{\circledR}$. A metodologia com relação $1: 1$ e os demais condutivímetros (WT-3000iCEL ${ }^{\circledR}$, CD-203 Phtek ${ }^{\circledR}$ e Hidroponia Digital CE ${ }^{\circledR}$ ) foram avaliados por índices estatísticos quanto a correlação, a precisão, a acurácia e os erros. A metodologia relação 1:1 apresentou precisão e correlação muito boa $\left(R^{2}=0,940 ; r=0,969\right)$, porém subestimou os valores (PBIAS $=19,952)$, sendo necessárias correções dos dados pelo modelo linear de regressão. Os condutivímetros WT-3000 iCEL ${ }^{\circledR}$ e portátil CD-203 Phtek ${ }^{\circledR}$ apresentaram, de maneira geral, desempenho insatisfatório pela maioria dos índices, devido ao limite das leituras de até $1,998 \mathrm{dS} \mathrm{m}^{-1}$ e a subestimativa dos dados. O Hidroponia Digital $\mathrm{CE}^{\circledR}$, obteve performance de boa a muito boa na metodologia referência, mas na relação 1:1 obteve uma maior subestimativa e apresentou índices insatisfatórios. Portanto, a metodologia e os condutivímetros alternativos subestimam a condutividade elétrica do solo e necessitam de correções.

Palavras-Chave: salinidade do solo, condutivímetros portáteis, fertirrigação, cultivos intensivos, índices de performance estatísticos.

\footnotetext{
1 Agrônomo, Doutor em Irrigação e Drenagem, Universidade Federal de Rondonópolis, Av. dos Estudantes, 5055 - Cidade Universitária, Rondonópolis - MT, 78736-900, e-mail: tonnyjasilva@ hotmail.com

${ }^{2}$ Eng. Agrícola, Universidade Federal de Rondonópolis, Av. dos Estudantes, 5055 - Cidade Universitária, Rondonópolis MT, 78736-900, e-mail: agricolanogueira@outlook.com

${ }^{3}$ Eng. Agrícola, Doutor em Engenharia de Sistemas Agrícolas, Rod. PA 140, 2428-4822, Tomé-Açu - PA, 68680-000, email: ad.pacheco@hotmail.com

${ }^{4}$ Zootecnista, Doutora em Agronomia (Solos e Nutrição de Plantas), Universidade Federal de Rondonópolis, Av. dos Estudantes, 5055 - Cidade Universitária, Rondonópolis - MT, 78736-900, e-mail: embonfim@ hotmail.com

5 Agrônomo, Doutor em Física Ambiental, Universidade Federal de Rondonópolis, Av. dos Estudantes, 5055 - Cidade Universitária, Rondonópolis - MT, 78736-900, e-mail: thiagof.duarte@ hotmail.com
} 


\title{
EVALUATION OF ALTERNATIVE METHOD AND CONDUCTIVIMETERS IN DETERMINING THE SOIL ELECTRICAL CONDUCTIVITY
}

\begin{abstract}
The aim was to evaluate, by means of statistical indexes, the estimate of the electrical conductivity of the soil by the methodology 1:1 ratio (soil: distilled water) and alternative conductivimeters, in a dystrophic Oxisol. The soil was subjected to the salinization process, with different concentrations of potassium chloride. It was adopted as a reference, the electrical conductivity of the solution extract soil measured by the mCA 150 Tecnal ${ }^{\circledR}$ conductivity meter. The $1: 1$ ratio methodology and the other conductivimeters (WT-3000iCEL ${ }^{\circledR}$, CD-203 Phtek $^{\circledR}$ and Hidroponia Digital CE ${ }^{\circledR}$ ) were evaluated by statistical indices regarding correlation, precision, accuracy and errors. The 1:1 ratio methodology showed Muito bom precision and correlation $\left(\mathrm{R}^{2}=0.940 ; \mathrm{r}=0.969\right)$, but underestimated the values (PBIAS $=19.952)$, requiring data corrections using the linear regression model. The conductivity meters WT-3000 iCEL ${ }^{\circledR}$ and portable CD-203 Phtek ${ }^{\circledR}$ presented, in general, poor performance by most indexes, due to the limit of the readings of up to $1.998 \mathrm{dS} \mathrm{m}^{-1}$ and the underestimation of the data. The Hidroponia Digital CE ${ }^{\circledR}$, obtained Good to Very Good performance in the reference methodology, but in the 1:1 ratio it obtained a greater underestimation and presented unsatisfactory indexes. Therefore, the methodology and alternative conductivimeters underestimate the electrical conductivity of the soil and need corrections.
\end{abstract}

Keywords: soil salinity, portable conductivimeters, fertigation, intensive cultivation, statistical performance indexes.

\section{INTRODUÇÃO}

Em cultivos intensivos e experimentos de base, com o uso de vasos ou recipientes em ambientes protegidos, o solo de cultivo está sujeito a riscos de salinização, devido à ausência de precipitações e às condições impostas, como o uso de adubações elevadas para culturas de alto rendimento, reuso de águas salobras ou salinas, estudos sobre tolerância a salinidade, pesquisas sobre o déficit hídrico, aplicação de tratamentos com doses elevadas de nutrientes em experimentos de fertilidade, entre outras condições desejadas pelos pesquisadores (NUNES JÚNIOR et al, 2017; KOETZ et al., 2019; SANTOS et al., 2020; SILVA et al., 2020; TIGKA; IPSILANTIS, 2020).

\footnotetext{
Nessas condições, os sais se acumulam nas camadas superiores do solo, comprometendo o desenvolvimento, a qualidade e a produtividade das culturas. Nesse sentido, o monitoramento da condutividade elétrica do solo é crucial, por ser a variável que representa a concentração total de sais solúveis no solo ou na água (DALIAKOPOULOS et al., 2016). Como os adubos apresentam potencial
}

de salinização, o monitoramento da condutividade elétrica do solo é usado como parâmetro para o manejo das adubações em fertirrigações de cultivos intensivos (OLIVEIRA et al., 2015; NUNES JÚNIOR et al., 2017), e por isso exige que sua determinação seja prática, rápida, confiável e de baixo custo.

O método considerado como referência na determinação da condutividade elétrica do solo utiliza o extrato da solução do solo e condutivímetros de bancada em laboratórios. Contudo, esse método utiliza amostras de solo entre 100 a 200 gramas de solo (Teixeira et al., 2017), um volume significativo para cultivos intensivos e experimentos em vasos ou recipientes pequenos $\left(1-13 \mathrm{dm}^{3}\right.$ volume de solo) (KOETZ et al., 2019; BONFIM et al., 2020; GUERRA et al., 2020; SANTOS et al., 2020; SILVA et al., 2020; TIGKA e IPSILANTIS, 2020).

Uma opção para essas condições de cultivo é o uso de metodologias e condutivímetros alternativos, para redução do volume da amostra, do tempo de determinação e dos custos, a fim de facilitar o monitoramento 
da condutividade elétrica do solo. Como metodologia alternativa, Richards (1954) propôs que diferentes relações terra: água destilada, podem ser usadas com boa precisão na determinação da condutividade elétrica do solo, se destacando a relação 1:1 por apresentar maior concentração de sais no meio aquoso, o que confere maiores variações de leituras. Desde então, diversos autores têm utilizado diferentes relações e observando especificidades para cada tipo de solo, como Abreu Junior et al. (2000) e Dias et al. (2005).

Contudo, para o uso de uma metodologia e condutivímetros alternativos, é necessário assegurar a confiabilidade dos dados determinados em relação ao método considerado referência. Nesse sentido, pode serem realizadas análises estatísticas que envolvam a estimativa da correlação, da precisão, da acurácia e dos erros, a fim de validar seus usos na determinação da condutividade elétrica do solo.

Diante do exposto, objetivou-se avaliar a estimativa da condutividade elétrica do solo pela metodologia com relação 1:1 (terra: água destila) e condutivímetros alternativos, em um Latossolo Vermelho distrófico.

\section{MATERIAL E MÉTODOS}

\section{Local dos ensaios e coleta e preparação do solo}

Os ensaios foram realizados no Instituto de Ciências Agrárias e Tecnológicas da Universidade Federal de Rondonópolis, Mato
Grosso, Brasil. O solo utilizado foi o Latossolo Vermelho distrófico (EMBRAPA, 2018), coletado na camada de 0,0-0,2m de profundidade, em área de Cerrado nativo do respectivo campus. Foi realizada a caracterização química e granulométrica do solo (EMBRAPA, 2017), que apresentou: $\mathrm{pH}$ $\left(\mathrm{CaCl}_{2}\right)=4,0 ; \mathrm{P}=1,4 \mathrm{mg} \mathrm{dm}^{-3} ; \mathrm{K}=23 \mathrm{mg} \mathrm{dm}^{-}$ 3; $\mathrm{Ca}=0,4 \mathrm{cmol}_{\mathrm{c}} \mathrm{dm}^{-3} ; \mathrm{Mg}=0,2 \mathrm{cmol}_{\mathrm{c}} \mathrm{dm}^{-3}$; $\mathrm{Al}=0,8 \mathrm{cmol}_{\mathrm{c}} \mathrm{dm}^{-3} ;$ M.O. $=27,1 \mathrm{~g} \mathrm{dm}^{-3} ; \mathrm{SB}=$ $0,7 \mathrm{cmol}_{\mathrm{c}} \mathrm{dm}^{-3} ; \mathrm{CTC}=6,8 \mathrm{cmol}_{\mathrm{c}} \mathrm{dm}^{-3} ; \mathrm{V}=$ 9,7\%; areia $=423 \mathrm{~g} \mathrm{~kg}^{-1}$; silte $=133 \mathrm{~g} \mathrm{~kg}^{-1}$; argila $=444 \mathrm{~g} \mathrm{~kg}^{-1}$.

O solo foi reservado em 32 unidades experimentais, que foram salinizadas com soluções de cloreto de potássio em diferentes concentrações, e deixado em casa de vegetação por um período de 20 dias de incubação em sacos plásticos, para obtenção de diferentes níveis de condutividade elétrica e para se ter homogeneidade na unidade.

Após a incubação, realizou-se a secagem à sombra do solo das unidades experimentais, para na sequência o solo ser peneirado em malha de $2 \mathrm{~mm}$ e proceder a determinação da condutividade elétrica em cada metodologia do estudo, no Laboratório de Solos e Nutrição Vegetal.

\section{Condutivímetros utilizados}

O condutivímetro de bancada mCA 150 Tecnal $^{\circledR}$ (Figura 1A) foi utilizado como referência, por permitir prévia calibração e ter compensação automática em relação à temperatura da amostra, o que garante confiabilidade e segurança na leitura.
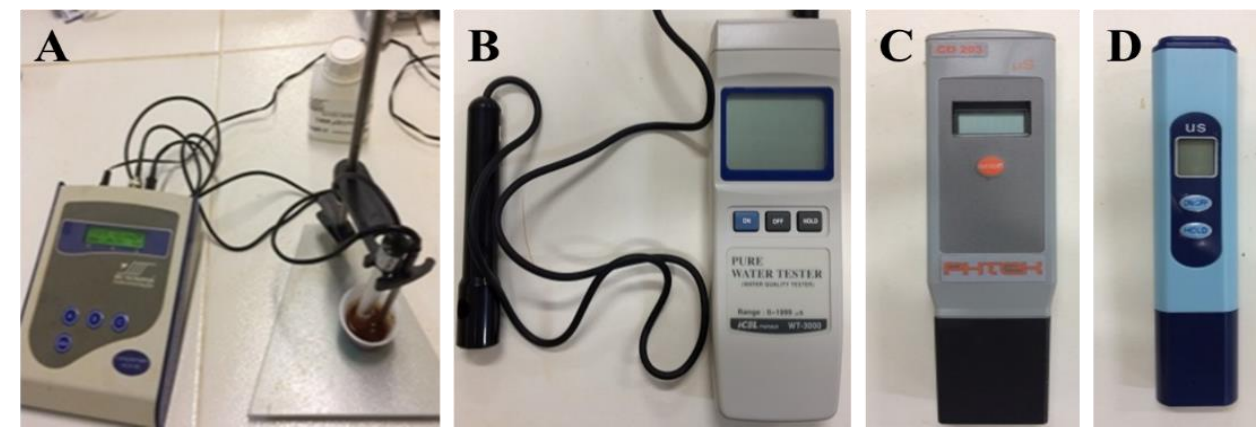

Figura 1. Condutivímetros de bancada mCA 150 Tecnal $^{\circledR}(\mathrm{A})$, semi-portátil WT-3000 iCEL ${ }^{\circledR}(\mathrm{B})$, portátil CD-203 Phtek $^{\circledR}(\mathrm{C})$, e Hidroponia Digital CE ${ }^{\circledR}(\mathrm{D})$. 
Os demais sensores alternativos foram escolhidos por permitirem maior praticidade por serem portáteis. Os condutivímetros semiportátil WT-3000 iCEL ${ }^{\circledR}$ (Figura 1B) e portátil CD-203 Phtek $^{\circledR}$ (Figura 1C) são comumente utilizados no monitoramento da condutividade elétrica da água de bacias hidrográficas e de estações de tratamento, e apresentam potencial para o monitoramento da condutividade elétrica para a mensuração da condutividade elétrica do solo, contudo são limitados a valores de até $1,998 \mathrm{dS} \mathrm{m}^{-1}$.

Por sua vez, o condutivímetro Hidroponia Digital $\mathrm{CE}^{\circledR}$ (Figura 1D) é utilizado no monitoramento de soluções nutritivas utilizadas na hidroponia e na fertirrigação, apresentando potencial para mensurar condutividade elétrica do solo por ter sido projetado para mensurar os níveis de interesse agrícola. Este sensor também a apresenta a vantagem de apresentar o menor custo de aquisição.

\section{Condutividade elétrica por meio do extrato da solução do solo}

A condutividade elétrica do solo por meio do extrato da solução foi realizada de acordo com a metodologia de Richards (1954) adaptada pela Embrapa (2017), inicialmente foram pesadas $100 \mathrm{~g}$ de solo e em recipientes apropriados adicionando-se água destilada até que a massa do solo apresentasse consistência de pasta, com aspecto brilhante ou espelhante, ou quando a pasta deslizasse suavemente na espátula. Posteriormente, a amostra ficou em repouso por 4 horas.

O solo foi colocado em funis de Büchner contendo papel de filtro e adaptados a kitassatos de $500 \mathrm{~mL}$. Aplicou-se a sucção com auxílio de um compressor de ar, por 40 minutos e coletou-se o extrato da solução do solo. Em seguida, foram mensuradas a condutividade elétrica do solo em cada condutivímetro, com temperatura da amostra de cerca de $25^{\circ} \mathrm{C}$.

\section{Condutividade elétrica por meio da relação 1:1 de solo:água destilada}

$\mathrm{Na}$ determinação da condutividade elétrica por meio da relação $1: 1$, as amostras foram preparadas com a mistura de $10 \mathrm{~cm}^{3}$ de terra fina seca ao ar com $10 \mathrm{~cm}^{3}$ de água destilada e agitação vigorosa por cinco minutos.

Após uma hora de repouso procedeu-se as leituras de condutividade elétrica do solo em cada condutivímetro, com a temperatura em cerca de $25^{\circ} \mathrm{C}$ (ABREU JUNIOR et al., 2000).

\section{Análises e avaliações dos dados}

O conjunto (método e condutivímetro) adotado como referência, e portanto, variável dependente $(\mathrm{y})$, foi a condutividade elétrica do solo do extrato de solução mensurada pelo condutivímetro de bancada mCA $150 \mathrm{Tecnal}^{\circledR}$. Os dados dos demais conjuntos foram considerados variáveis independentes (x), a fim a avaliar sua predição nos valores de condutividade elétrica do solo.

Os dados foram submetidos a análise de regressão, a $5 \%$ de probabilidade, para obtenção do modelo linear regressão. A partir do modelo, foi avaliada a qualidade da performance (Tabela 1) de cada conjunto (método e condutivímetro) a partir dos coeficientes, erros e índices estatísticos, sendo eles: coeficiente de determinação $\left(\mathrm{R}^{2}\right)$, correlação de Pearson (r), erro médio (MAE), erro médio absoluto (EMAX), raiz do erro médio quadrático (RMSE), Índice de Concordância de Willmott (d) (WILLMOTT, 1981), Índice de Confiança (c) (CAMARGO e SENTELHAS, 1997), eficiência de Nash-Sutcliffe (NSE) (NASH e SUTCLIFFE, 1970), o viés percentual (PBIAS) (YAPO et al., 1996) e o Akaike Information Criterion (AIC) (AKAIKE, 1974). Todas as análises foram realizadas no software $\mathrm{R}$ (version 3.6.2). 
Tabela 1. Critérios de avaliação de desempenho qualitativo para os índices estatísticos dos métodos de determinação de área foliar (adaptados de MORIASI et al., 2015; e de PACHECO et al, 2020).

\begin{tabular}{ccccc}
\hline \multirow{2}{*}{$\begin{array}{c}\text { Índice } \\
\text { Estatísticos }\end{array}$} & \multicolumn{4}{c}{ Performance do modelo } \\
\cline { 2 - 5 } $\mathrm{R}^{2}$ & Muito bom & Bom & Satisfatório & Insatisfatório \\
\hline $\mathrm{r}$ & $>0,85$ & $0,75<\mathrm{R}^{2} \leq 0,85$ & $0,60<\mathrm{R}^{2} \leq 0,75$ & $\leq 0,60$ \\
$\mathrm{~d}$ & $>0,85$ & $0,60<\mathrm{r} \leq 0,85$ & $0,40<\mathrm{r} \leq 0,60$ & $\leq 0,40$ \\
$\mathrm{c}$ & $>0,90$ & $0,85<\mathrm{d} \leq 0,90$ & $0,75<\mathrm{d}<0,85$ & $\leq 0,75$ \\
$\mathrm{NSE}$ & $>0,84$ & $0,66<\mathrm{c} \leq 0,84$ & $0,61<\mathrm{c} \leq 0,66$ & $\leq 0,61$ \\
PBIAS $(\%)$ & $< \pm 5$ & $0,70<\mathrm{NSE} \leq 0,80$ & $0,50<\mathrm{NSE} \leq 0,70$ & $\leq 0,50$ \\
& & $\pm 5 \leq \mathrm{PBIAS} \leq \pm 10$ & $\pm 10 \leq \mathrm{PBIAS} \leq \pm 15$ & $\geq \pm 15$ \\
\hline
\end{tabular}

\section{RESULTADOS E DISCUSSÃO}

A metodologia alternativa com a relação 1:1 (solo:água destilada) apresentou precisão e correlação classificada como muito bom na determinação da condutividade elétrica do solo, ao considerar os valores de coeficiente de determinação $\left(\mathrm{R}^{2}=0,940\right)$ e a correlação de Pearson $(\mathrm{r}=0,969)$, respectivamente. Contudo, o método obteve os maiores erros (MAE $=1,061 ;$ EMAX $=2,579 ;$ RMSE $=1.280$ ), quando comparado com a mensuração pelos outros conjuntos de metodologias do estudo (Figura 2; Tabela 2).

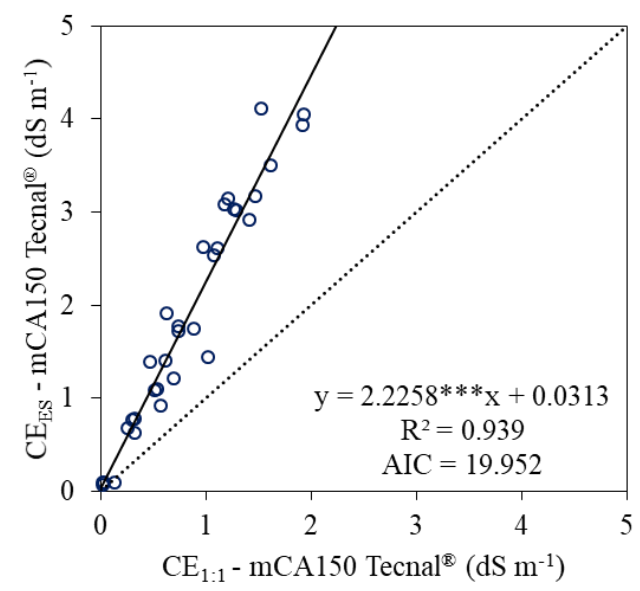

Figura 2. Condutividade elétrica do extrato da solução $\left(\mathrm{CE}_{\mathrm{ES}}\right)$ determinada e estimada a partir da condutividade elétrica da relação 1:1 $\left(\mathrm{CE}_{1: 1}\right)$, ambas mensuradas pelo condutivímetro de bancada mCA150 da Tecnal ${ }^{\circledR}$.*** modelo significativo a $0,01 \%$.

A eficiência do modelo apresentou-se insatisfatório (NSE $=-0,090)$, devido a subestimativa (PBIAS $=19,952$ ) observada dos valores da condutividade elétrica do solo pela relação $1: 1$, quando comparada com o extrato da solução, sendo necessária a correção com o uso de equação. O modelo gerado apresentouse adequado para as correções, pois obteve um baixo valor Akaike Information Criterion (AIC = 19,952) (Figura 2; Tabela 3).

A subestimativa observada na condutividade elétrica da relação 1:1, deve-se a uma maior diluição dos sais da solução do solo, quando comparado com o extrato da solução (método adotado como referência). Dias et al. (2005) enfatizam que a estimativa da condutividade elétrica por meio de relações solo:água destilada apresenta como vantagens rapidez na execução e menores custos de operação por dispensar o uso de equipamentos para obtenção da solução do solo.

Contudo, os autores ressaltam a importância de se obterem equações de correção para o tipo de solo a ser trabalhado. 
Tabela 2. Coeficiente de determinação $\left(\mathrm{R}^{2}\right)$, erro médio (MAE), erro máximo (RMSE) e correlação de Pearson (r) para os métodos e condutivímetros na determinação da condutividade elétrica do solo por meio do extrato da solução.

\begin{tabular}{|c|c|c|c|c|c|c|}
\hline Método & Condutivímetro & $\mathrm{R}^{2}$ & MAE & EMAX & RMSE & $\mathrm{r}$ \\
\hline \multirow{5}{*}{$\begin{array}{l}\text { Extrato de saturação } \\
\text { (CEes) }\end{array}$} & \multirow{2}{*}{ WT-3000 iCEL ${ }^{\circledR}$} & 0.705 & 0.595 & 2.111 & 0.863 & 0.840 \\
\hline & & Satisfatório & -- & -- & -- & Bom \\
\hline & \multirow{2}{*}{ CD-203 Phtek ${ }^{\circledR}$} & 0.614 & 0.663 & 2.421 & 0.972 & 0.784 \\
\hline & & Satisfatório & -- & -- & -- & Bom \\
\hline & $\begin{array}{l}\text { Hidroponia Digital } \\
\qquad \mathrm{CE}^{\circledR}\end{array}$ & $\begin{array}{c}0.979 \\
\text { Muito bom }\end{array}$ & $\begin{array}{c}0.211 \\
\text { Melhor } \\
\text { resultado }\end{array}$ & $\begin{array}{c}0.830 \\
\text { Melhor } \\
\text { resultado }\end{array}$ & $\begin{array}{c}0.323 \\
\text { Melhor } \\
\text { resultado }\end{array}$ & $\begin{array}{c}0.989 \\
\text { Muito bom }\end{array}$ \\
\hline \multirow{7}{*}{$\begin{array}{c}\text { Relação de volume } \\
1: 1 \\
\text { (solo:água destilada) }\end{array}$} & mCA150 Tecnal $^{\circledR}$ & $\begin{array}{l}0.940 \\
\text { Muito bom }\end{array}$ & $\begin{array}{c}1.061 \\
\text { Pior resultado }\end{array}$ & $\begin{array}{c}2.579 \\
\text { Pior resultado }\end{array}$ & $\begin{array}{c}1.280 \\
\text { Pior resultado }\end{array}$ & $\begin{array}{c}0.969 \\
\text { Muito bom }\end{array}$ \\
\hline & \multirow{2}{*}{ WT-3000 iCEL ${ }^{\circledR}$} & 0.910 & 0.918 & 2.111 & 1.109 & 0.954 \\
\hline & & Muito bom & -- & -- & -- & Muito bom \\
\hline & \multirow{2}{*}{ CD-203 Phtek ${ }^{\circledR}$} & 0.931 & 0.886 & 2.111 & 1.080 & 0.965 \\
\hline & & Muito bom & -- & -- & -- & Muito bom \\
\hline & \multirow{2}{*}{$\begin{array}{l}\text { Hidroponia Digital } \\
\mathrm{CE}^{\circledR}\end{array}$} & 0.940 & 0.869 & 2.190 & 1.082 & 0.969 \\
\hline & & Muito bom & -- & -- & -- & Muito bom \\
\hline
\end{tabular}

Tabela 3. Índice de concordância de Willmott (d), índice de performance de Camargo e Sentelhas (c), coeficiente de eficiência do modelo (NSE), viés percentual (PBIAS) e Akaike Information Criterion (AIC) para os métodos e condutivímetros na determinação da condutividade elétrica do solo por meio do extrato da solução.

\begin{tabular}{|c|c|c|c|c|c|c|}
\hline Método & Condutivímetro & d & $\mathrm{c}$ & NSE & PBIAS & AIC \\
\hline \multirow{3}{*}{$\begin{array}{l}\text { Extrato de saturação } \\
\text { (CEes) }\end{array}$} & WT-3000 iCEL ${ }^{\circledR}$ & $\begin{array}{c}0.808 \\
\text { Satisfatório }\end{array}$ & $\begin{array}{l}0.755 \\
\text { Bom }\end{array}$ & $\begin{array}{c}0.504 \\
\text { Satisfatório }\end{array}$ & $\begin{array}{c}-25.200 \\
\text { Insatisfatório }\end{array}$ & $\begin{array}{c}70.756 \\
--\end{array}$ \\
\hline & CD-203 Phtek ${ }^{\circledR}$ & $\begin{array}{c}0.752 \\
\text { Satisfatório }\end{array}$ & $\begin{array}{l}0.680 \\
\text { Bom }\end{array}$ & $\begin{array}{c}0.371 \\
\text { Insatisfatório }\end{array}$ & $\begin{array}{c}-28.900 \\
\text { Insatisfatório }\end{array}$ & $\begin{array}{c}79.397 \\
\text { Pior resultado }\end{array}$ \\
\hline & $\begin{array}{l}\text { Hidroponia Digital } \\
\mathrm{CE}^{\circledR}\end{array}$ & $\begin{array}{c}0.979 \\
\text { Muito bom }\end{array}$ & $\begin{array}{c}0.979 \\
\text { Muito bom }\end{array}$ & $\begin{array}{c}0.931 \\
\text { Muito bom }\end{array}$ & $\begin{array}{l}-9.700 \\
\text { Bom }\end{array}$ & $\begin{array}{l}-13.825 \\
\text { Melhor resultado }\end{array}$ \\
\hline \multirow{4}{*}{$\begin{array}{c}\text { Relação de volume } \\
1: 1 \\
\text { (terra:água destilada) }\end{array}$} & mCA150 Tecnal $^{\circledR}$ & $\begin{array}{c}0.677 \\
\text { Insatisfatório }\end{array}$ & $\begin{array}{l}0.798 \\
\text { Bom }\end{array}$ & $\begin{array}{c}-0.090 \\
\text { Insatisfatório }\end{array}$ & $\begin{array}{c}-55.800 \\
\text { Insatisfatório }\end{array}$ & $\begin{array}{c}19.952 \\
--\end{array}$ \\
\hline & WT-3000 iCEL ${ }^{\circledR}$ & $\begin{array}{c}0.742 \\
\text { Insatisfatório }\end{array}$ & $\begin{array}{l}0.821 \\
\text { Bom }\end{array}$ & $\begin{array}{c}0.182 \\
\text { Insatisfatório }\end{array}$ & $\begin{array}{c}-48.400 \\
\text { Insatisfatório }\end{array}$ & $\begin{array}{c}32.909 \\
--\end{array}$ \\
\hline & CD-203 Phtek ${ }^{\circledR}$ & $\begin{array}{c}0.747 \\
\text { Insatisfatório }\end{array}$ & $\begin{array}{l}0.834 \\
\text { Bom }\end{array}$ & $\begin{array}{c}0.225 \\
\text { Insatisfatório }\end{array}$ & $\begin{array}{c}-46.700 \\
\text { Insatisfatório }\end{array}$ & $\begin{array}{c}24.363 \\
--\end{array}$ \\
\hline & $\begin{array}{c}\text { Hidroponia Digital } \\
\mathrm{CE}^{\circledR}\end{array}$ & $\begin{array}{c}0.741 \\
\text { Insatisfatório }\end{array}$ & $\begin{array}{l}0.835 \\
\text { Bom }\end{array}$ & $\begin{array}{c}0.221 \\
\text { Insatisfatório }\end{array}$ & $\begin{array}{c}-45.700 \\
\text { Insatisfatório }\end{array}$ & $\begin{array}{c}19.987 \\
--\end{array}$ \\
\hline
\end{tabular}

Os condutivímetros semi-portátil WT3000 iCEL $^{\circledR}$ (Figura 3A) e portátil CD-203 Phtek $^{\circledR}$ (Figura 4A) apresentaram coeficientes de determinação e correlações de Pearson de satisfatórios a muito bom.

No entanto, os demais coeficientes e índices avaliados foram, de maneira geral, insatisfatórios, devido ao limite das leituras dos sensores de até $1,998 \mathrm{dS} \mathrm{m}^{-1}$ e pela subestimativa da condutividade elétrica (Tabelas 2 e 3). Na determinação da condutividade elétrica pela relação 1:1, os sensores apresentaram os índices de performance bom, devido aà menor condutividade das amostras que possibilitaram um maior número de medidas em seus intervalos de leitura (Tabelas 2 e 3; Figuras 3B e 4B). 

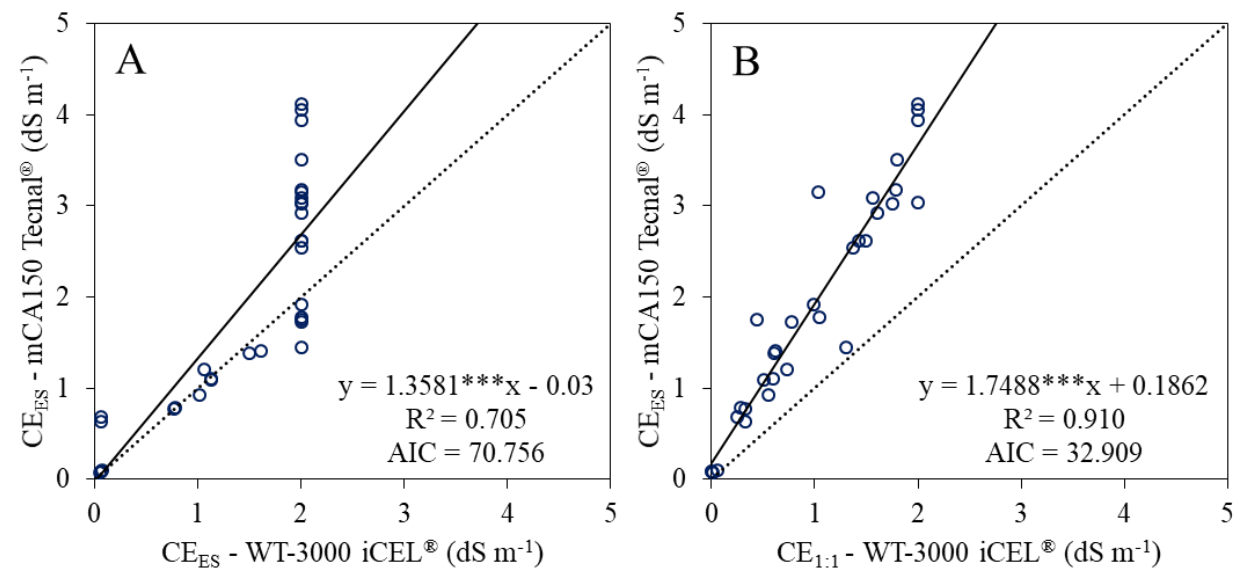

Figura 3. Condutividade elétrica do extrato da solução (CEES) pelo condutivímetro de bancada mCA150 da Tecnal ${ }^{\circledR}$ determinada e estimada a partir da condutividade elétrica do solo do extrato da solução (A) e da relação 1:1 (CE1:1) (B), ambas mensuradas pelo sensor semi-portátil WT-3000 iCel ${ }^{\circledR}$. *** modelos significativos a $0,01 \%$.
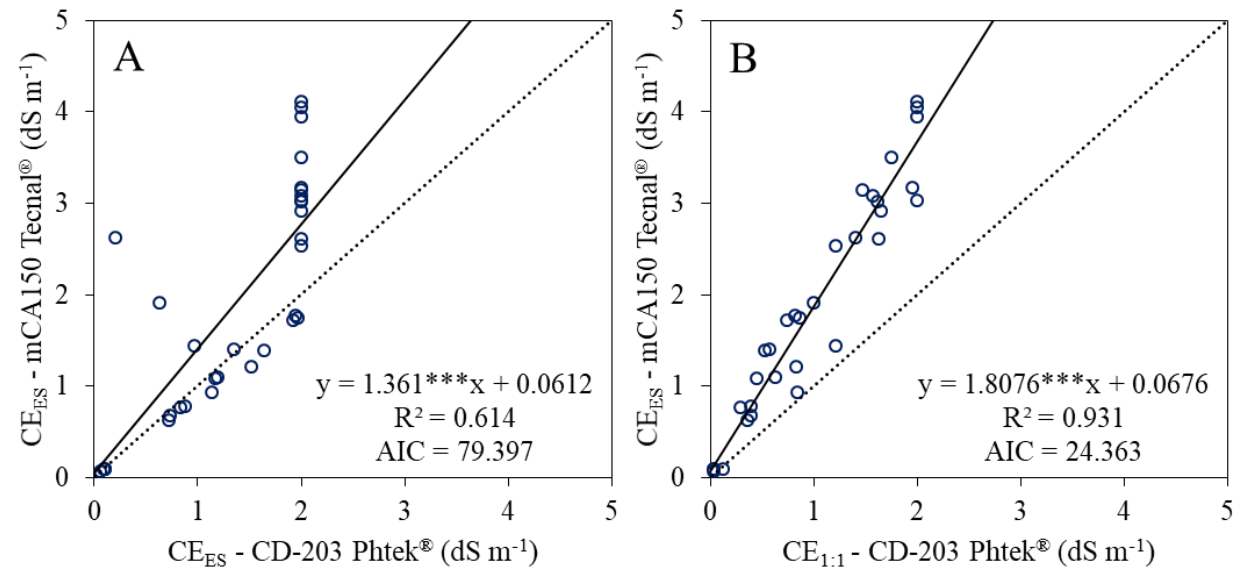

Figura 4. Condutividade elétrica do extrato da solução (CEES) pelo condutivímetro de bancada mCA150 da Tecnal ${ }^{\circledR}$ determinada e estimada a partir da condutividade elétrica do solo do extrato da solução (A) e da relação 1:1 $\left(\mathrm{CE}_{1: 1}\right)(\mathrm{B})$, ambas mensuradas pelo sensor portátil CD-203 Phtek $^{\circledR}$. *** modelos significativos a $0,01 \%$.

O condutivímetro portátil Hidroponia Digital $\mathrm{CE}^{\circledR}$ sobressaiu-se, em relação aos demais condutivímetros alternativos, na mensuração da condutividade elétrica do solo no extrato de solução, obtendo coeficientes e índices de performance de bons a muito bons, com menores erros e viés percentual que indica menor subestimativa (Tabelas 2 e 3; Figura
5A). Na condutividade elétrica de relação 1:1, o sensor apresentou coeficiente de determinação e correção de Pearson muito bons, contudo no desempenho dos demais coeficientes avaliados apresentou resultados insatisfatórios (Tabela 2 e 3; Figura 5B) devido ao aumento da subestimativa incrementada pelas características do método (Figura 2). 

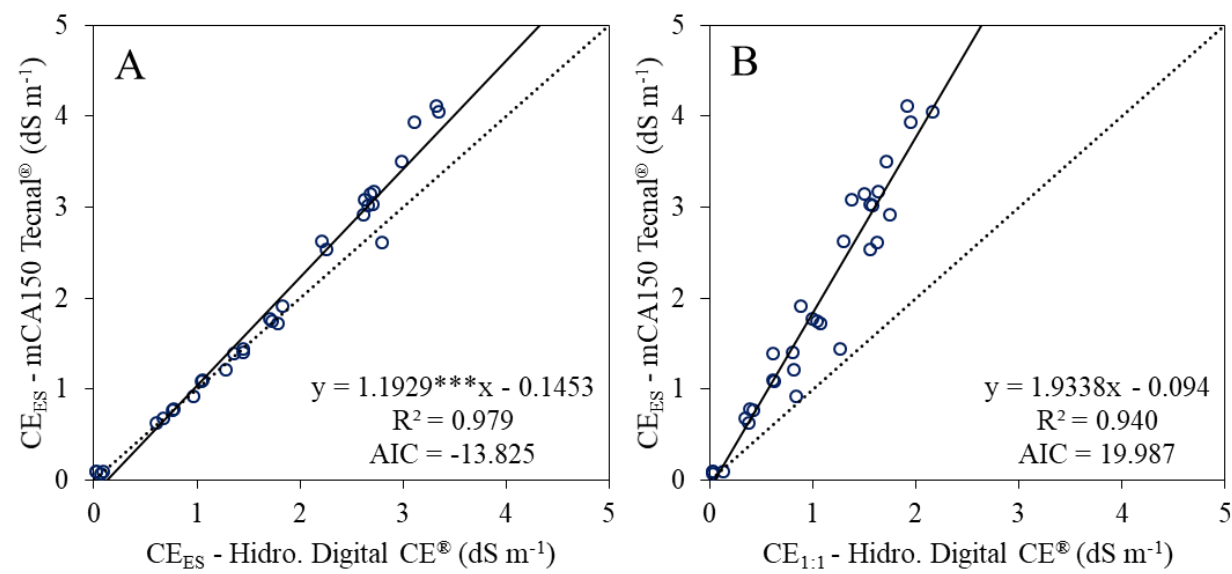

Figura 5. Condutividade elétrica do extrato da solução (CEES) pelo condutivímetro de bancada mCA150 da Tecnal ${ }^{\circledR}$ determinada e estimada a partir da condutividade elétrica do solo do extrato da solução (A) e da relação 1:1 (CE1:1) (B), ambas mensuradas pelo sensor portátil Hidroponia Digital $\mathrm{CE}^{\circledR}$. $* * *$ modelos significativos a $0,01 \%$.

A metodologia de relação 1:1 e o condutivímetro Hidroponia Digital $\mathrm{CE}^{\circledR}$ apresentaram-se adequados ao monitoramento da condutividade elétrica do solo. Por sua vez, os condutivímetros semi-portátil WT-3000 iCel ${ }^{\circledR}$ e portátil CD-203 Phtek ${ }^{\circledR}$ são limitados ao limite de leitura de até $1,998 \mathrm{dS} \mathrm{m} \mathrm{m}^{-1}$, sendo recomendado apenas para o monitoramento nos cultivos de culturas sensíveis a salinidade, as quais apesentam perdas do rendimento relativo a partir de $1 \mathrm{dS} \mathrm{m}^{-1}$ (DIAS et al., 2017).

Vale ressaltar, que os dados da metodologia e dos condutivímetros alternativos estudados, necessitam de correção para a estimativa da condutividade elétrica do extrato de saturação. E que, para outros tipos de solo é recomendado a confecção de curvas de calibrações específicas.

\section{CONCLUSÕES}

A metodologia e os condutivímetros alternativos subestimam a condutividade elétrica do solo e necessitam de correções por modelos lineares de regressão.

A metodologia alternativa, relação $1: 1$, apresentou precisão e correlação muito boa, porém subestimou os valores, sendo necessário correções dos dados pelo modelo linear de regressão.

Os condutivímetros WT-3000 iCEL ${ }^{\circledR} \mathrm{e}$ portátil CD-203 Phtek $^{\circledR}$ apresentaram, de maneira geral, desempenho insatisfatórios pela maioria dos índices.

O Hidroponia Digital $\mathrm{CE}^{\circledR}$, obteve performance de boa a muito boa na metodologia referência, mas na relação 1:1 obteve uma maior subestimativa e apresentou índices insatisfatórios.

\section{AGRADECIMENTOS}

Os autores agradecem ao Conselho Nacional de Desenvolvimento Científico e Tecnológico (CNPq) e à Coordenação de Aperfeiçoamento de Pessoal de Nível Superior (CAPES) pela concessão de bolsas de estudo aos autores.

\section{REFERÊNCIAS BIBLIOGRÁFICA}

ABREU JUNIOR, C. H.; MURAOKA, T.; LAVORANTE, A. F.; ALVAREZ V., F. C. Condutividade elétrica, reação do solo e acidez potencial em solos adubados com composto de lixo. Revista Brasileira de Ciência do Solo, v. 24, n. 3, p.635-647, 2000. https://doi.org/10.1590/S010006832000000300016

AKAIKE, H. A new look at the statistical model identification. IEEE Transactions on Automatic Control, v. 19, n. 6, p. 716-723, 
1974.

http://dx.doi.org/10.1109/TAC.1974.1100705

BONFIM, G. V.; SANTOS, M. D. S.; AZEVEDO, B. M.; CARVALHO, A. C. P. P.; FERNANDES, C. N. V. Irrigation intervals in the production of ornamental pineapple in pots. Revista Caatinga, v. 33, n. 1, p. 226-235, $2020 . \quad \mathrm{http} / / / \mathrm{dx}$.doi.org/10.1590/198321252020v33n124rc

CAMARGO, A.P.; SENTELHAS, P.C. Avaliação do desempenho de diferentes métodos de estimativa da evapotranspiração potencial no Estado de São Paulo, Brasil. Revista Brasileira de Agrometeorologia, v. 5, n. 1, p. 89-97, 1997.

DALIAKOPOULOS, I. N.; TSANIS, I. K.; KOUTROULIS, A.; KOURGIALAS, N. N.; VAROUCHAKIS, A. E.; KARATZAS, G. P.; RITSEMA, C. J. The threat of soil salinity: A European scale review. Science of the Total Environment, v. 573, p. 727-739, 2016. http://dx.doi.org/10.1016/j.scitotenv.2016.08.1 77

DIAS, N. S.; BLANCO, FLÁVIO F.; SOUZA, E. R.; FERREIRA, J. F. S.; SOUSA NETO, O. N.; QUEIROZ, I. S. R. de. Efeitos dos sais na planta e tolerância das culturas à salinidade. Gheyi, H. R.; Dias, N. da S.; Lacerda, C. F. de; Gomes Filho, E. (ed.). Manejo da salinidade na agricultura: Estudos básicos e aplicados. Fortaleza: INCTSal, 2017. cap. 11, p. 151-162.

DIAS, N. S.; DUARTE, S. N.; GHEYI, H. R.; MEDEIROS, J. F.; SOARES, T. M. Manejo da fertirrigação e controle da salinidade do solo sob ambiente protegido, utilizando-se extratores de solução do solo. Revista Brasileira de Engenharia Agrícola e Ambiental, v.9, p.496-504, 2005. https://doi.org/10.1590/S1415-

43662005000400009

TEIXEIRA, DONAGEMMA, G. K.; FONTANA, A.; TEIXEIRA, W. G. Manual de métodos de análise de solo. $3^{\mathrm{a}}$ ed. rev. e ampl. Brasília: Embrapa, 2017. 574 p.

SANTOS, H. G.; JACOMINE, P. K. T.; ANJOS, L. H. C.; OLIVEIRA, V. A.; LUMBRERAS, J. F.; COELHO, M. R.; ALMEIDA, J. A.; ARAUJO FILHO, J. C.; OLIVEIRA, J. B.; CUNHA, T. J. F. Sistema Brasileiro de Classificação de Solos. $5^{\text {a }}$ ed. rev. e ampl. Brasília: Embrapa, 2018. 356p.

GUERRA, A. M. N. M.; SILVA, M. G. M.; EVANGELISTA, R. S. growth environment and pot volume affect biomass and essential oil production of basil. Revista Caatinga, v. 33, n. 1, p. 135-141, 2020. http://dx.doi.org/10.1590/198321252020v33n115rc

KOETZ, M.; PORTO, R. A.; BONFIMSILVA, E. M.; POLYZEL, A. C.; SILVA, T. J. A. Effects of nitrogen fertilization and water replacement level on the production of gladiolus corms in a protected environment. Journal of Agricultural Science, v. 11, n. 9, 2019. https://doi.org/10.5539/jas.v11n9p234

MORIASI, D. N.; GITAU, M. W.; PAI, N.; DAGGUPATI, P. Hydrologic and water quality models: Performance measures and evaluation criteria. Transactions of the ASABE, v. 58, n. 6, p. 1763-1785, 2015. https://doi.org/10.13031/trans.58.10715

NASH, J. E.; SUTCLIFFE, J. V. River flow forecasting through conceptual models part IA discussion of principles. Journal of hydrology, v. 10, n. 3, p. 282-290, 1970. https://doi.org/10.1016/0022-1694(70)90255-6

NUNES JÚNIOR, E. S.; MEDEIROS; J. F., OLIVEIRA; F. A.; LIMA, L. A.; BEZERRA, F. M. S.; ALVES, R. C. Nitrogen and potassium fertigation in bell pepper cultivated in greenhouse using fertigation managements.

Revista Brasileira de Engenharia Agrícola e Ambiental, v. 21, n. 3, p. 186-190, 2017. https://doi.org/10.1590/1807-

1929/agriambi.v21n3p186-190 
OLIVEIRA, F. A.; DUARTE, S. N.; MEDEIROS, J.F.; DIAS, N. S.; OLIVEIRA, M. K. T.; SILVA, R. C. P.; LIMA, K. S. 2015. Nutrição mineral do pimentão submetido a diferentes manejos de fertirrigação. Horticultura Brasileira, v. 33, n.2, p. 216$223 . \quad \mathrm{http}: / / \mathrm{dx}$.doi.org/10.1590/S0102053620150000200013

PACHECO, A. B.; NASCIMENTO, J. G.; MOURA, L. B.; LOPES, T. R.; DUARTE, S. N.; COELHO, R. D.; MARQUES, P. A. A. Non-destructive and Destructive Methods to Determine the Leaf Area of Zucchini. Journal of Agricultural Studies, v. 8, n. 3, p. 295-309, 2020. https://doi.org/10.5296/jas.v8i3.16299

RICHARDS, L.A. Diagnosis and improvement of saline and alkali soils. Agriculture Handbook, 60. Washington: United States Departament of Agriculture, 1954. 160p.

SANTOS, S. T.; OLIVEIRA, F. A.; OLIVEIRA, G. B. S.; SÁ, F. V. S.; COSTA, J. P. B. M.; FERNANDES, P. D. Photochemical efficiency of basil cultivars fertigated with salinized nutrient solutions. Revista Brasileira de Engenharia Agrícola e Ambiental, v. 24, n. , p. 320-325, 2020. http://dx.doi.org/10.1590/1807-

1929/agriambi.v24n5p320-325

SILVA, C. T. R.; BONFIM-SILVA, E. M.; SILVA, T. J. A.; PINHEIRO, E. A. R.; JOSÉ, J. V.; FERRAZ, A. P. F. Yield Component Responses of the Brachiaria brizantha Forage Grass to Soil Water Availability in the Brazilian Cerrado. Agriculture, v. 10, n. 1, p. $1-17$, 2020 .

https://doi.org/10.3390/agriculture10010013

TIGKA, T.; IPSILANTIS, I. Effects of sand dune, desert and field arbuscular mycorrhizae on lettuce (Lactuca sativa, L.) growth in a natural saline soil. Scientia Horticulturae, v. 264, p. $109191, \quad 2020$. https://doi.org/10.1016/j.scienta.2020.109191

WILLMOTT, C. J. On the validation of models. Physical geography, v. 2, n. 2, p. 184-194, 1981. https://doi.org/10.1080/02723646.1981.10642 213

YAPO P. O.; GUPTA H. V.; SOROOSHIAN $\mathrm{S}$. Automatic calibration of conceptual rainfallrunoff models: sensitivity to calibration data. Journal of Hydrology. v. 181 n. 4. p. 23-48, $1996 . \quad$ https://doi.org/10.1016/00221694(95)02918-4 